\title{
Rate Control for DS-CDMA Channels Using Power Control and Short Orthogonal Pseudo Random Codes
}

\author{
Alejandra Mercado and K.J. Ray Liu \\ Department of Electrical and Computer Engineering \\ University of Maryland \\ College Park, MD 20742 \\ kjrliu@eng.umd.edu
}

\begin{abstract}
Multimedia applications are increasingly seen in wireless service networks. The higher channel rate requirements of certain multimedia services, such as video, places constraints on wireless resources, which must provide high channel rates in the presence of high interference and noise. We propose to increase the channel rate of high rate multimedia users by controlling the effective signal to interference and noise ratio (SINR) for those users, and by breaking individual data streams into smaller substreams which each have lower capacity requirements.
\end{abstract}

Keywords-wireless networks, multimedia, rate control

\section{INTRODUCTION}

Consider a DS-CDMA system which splits each multimedia user's data stream into several independently coded substreams [1]. Each of the user's substreams are spread with short orthogonal pseudo random sequences (SOPRaS). The number of substreams for each user is selected to maximize the capacity for each user. Effectively, we transform a user channel that has high rate requirement, and would thus require high SINR, into a virtual set of lower rate channels.

Controlling the SINR is a result of the automatic power control,[2]. In wireless power control, the SINR levels which are low enough to allow for convergence to a positive power vector are called feasible SINR levels. Our goal is to increase the feasible set of SINR levels in a DS-CDMA system which uses power control and space-time diversity, [3].

We strive to assign the highest feasible SINR levels to all multimedia users. The feasibility of a given set of SINR is tested by the invertibility of the system gain matrix [4]. It can be shown that, under certain circumstances, the feasibility constraint for the power control is a separable problem from how many substreams are assigned to each user, and the SINR allocation for each user's substream. We can provide a method to find feasible SINR allocation for each user, and given that, we can find the optimum number of substreams per user and the optimum SINR allocation for each substream. By optimum, we mean that our solution maximizes the user's channel capacity.

\section{SySTEM MODEL}

Fig. 1 illustrates the transmitter for the proposed system. The left part of Fig. 1 shows how the data sequence are formed. Each user, $k$, has $r_{k}$ substreams. Each data stream, $i$, of user $k$ is turned into an analogue signal, $d_{k, i}(t)$. Then each substream is spread with a SOPRaS code, $c_{i}$. The SOPRaS spread substreams, $e_{k, i}$, are then added together and spread with user $k$ 's pseudo-random spreading sequence (PRSS), $f_{k}$. The output of that is then modulated and transmitted. The effect of using SOPRaS is turning a single user, $k$, into $r_{k}$ virtual users. Thus, the signal before modulation for user $k$ is:

$$
s_{k}(t)=\sqrt{P_{k}} \sum_{i=1}^{r_{k}} \underbrace{d_{k, i}(t) c_{i}(t)}_{e_{k, i}} f_{k}(t)
$$

where $c_{i}(\cdot)$ is the SOPRaS pulse train and $f_{k}(\cdot)$ is the PRSS pulse train.

Fig. 2 shows the proposed receiver. The base station has $D$ antennas, and RAKE receivers for space-time diversity. The signals from all multipaths from all users are received along with white Gaussian noise, and then they are demodulated. The output of the antenna arrays, $\mathbf{x}$, is a $D \times 1$ vector:

$$
\mathbf{x}(t)=\sum_{k=1}^{M} \sum_{l=1}^{L_{k}} s_{k}\left(t-\tau_{k, l}\right) G_{k, l} \mathbf{a}_{k, l} e^{-j \omega_{c} \tau_{k, l}}+\mathbf{n}(t)
$$

where $G_{k, l}, \mathbf{a}_{k, l}$, and $\tau_{k, l}$ are the pathloss, array response vector, and the delay of the $l^{t h}$ multipath of the $k^{t h}$ user, respectively.

Vector $\mathbf{x}$ passes through the despreading block corresponding to the $i^{\text {th }}$ SOPRaS for the $k^{t h}$ user, resulting in $\mathbf{y}_{(l) i}^{k}$. Assuming coherent reception and an accurate estimate of the $l^{\text {th }}$ multipath delay of user $k$, we have that

$$
\begin{aligned}
\mathbf{y}_{(l) i}^{k}(t)= & \frac{1}{\sqrt{T_{b}}} \int_{\tau-\tau_{k, l}}^{\tau-\tau_{k, l}+T_{b}} \mathbf{x}(v) c_{i}\left(v-\tau_{k, l}\right) \\
= & f_{k}\left(v-\tau_{k, l}\right) d v \\
& +{ }_{P_{i}^{k}}^{k} G_{k, l} d_{(l) i}^{k}\left(n T_{b}\right) \mathbf{a}_{k, l} e^{-j \omega_{c} \tau_{k, l}}+\mathbf{S I}_{(l) i}^{k} \\
& +\mathbf{M} \mathbf{A I}_{(l) i}^{k}+\mathbf{n}_{(l) i}^{k}
\end{aligned}
$$

for $t \in\left[\tau, \tau+T_{b}\right)$, where $\mathbf{n}_{k, l}(\cdot)$ is the thermal noise after the despreading effect. $\mathbf{M A I}_{k, l}(\cdot)$ is the distortion produced by multiple access interference, and $\mathbf{S I}_{k, l}(\cdot)$ is the interference produced by user $k$ 's multipaths.

The space-time weights are applied to $\mathrm{y}_{(l) i}^{k}$ producing a scalar, $z_{(l) i}^{k}=\left(\mathbf{w}_{(l)}^{k}\right)^{H} \mathbf{y}_{(l) i}^{k}$. Obtaining the space-time 


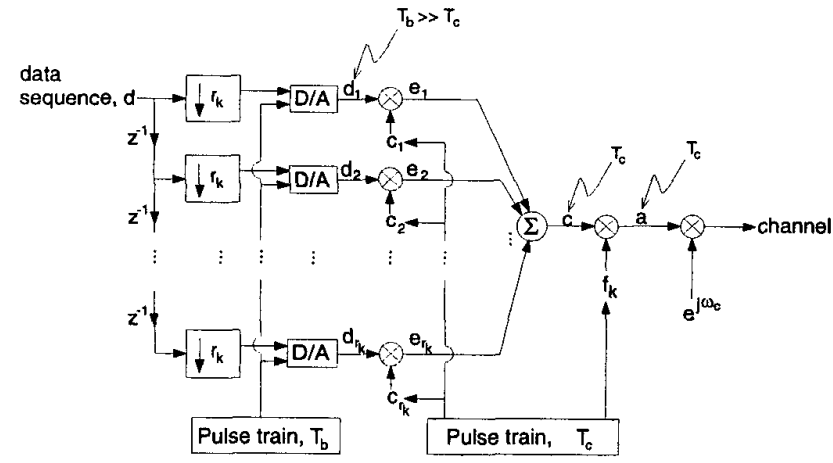

Fig. 1. Proposed transmitter for user $k$. There are $r_{k}$ substreams. Substream $d_{j}$ is spread with a SOPRaS code, $c_{j}$. All substreams are then added and then spread with the user spreading code, $f_{k}$.

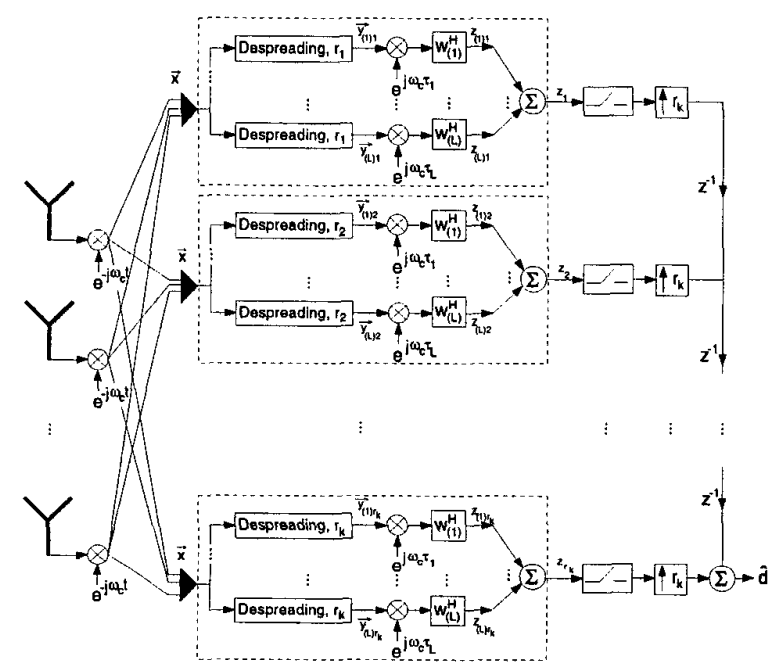

Fig. 2. Proposed receiver. The antenna array has $D$ elements. Each vector portrayed before and after the spreading boxes, $\mathbf{x}_{(j) i}$ and $\mathbf{y}_{(j) i}$ are $D$ dimensional. Each dotted box represents one of users $k$ 's $r_{k}$ substreams. Each substream box has $L$ multipath fingers.

weights and estimating the multipath delays has been addressed in [5]. The results are then added producing the output of the space-time diversity receiver, $z_{i}^{k}(t)$, which is sampled and then the original sequence is reconstructed from all the substreams.

\section{RATE CONTROL}

We control the channel rate for any user, $k$ by controlling the number of substreams and the channel rate of each of its $r_{k}$ substreams. To this end, we focus on $z_{i}^{k}(t)$. Let

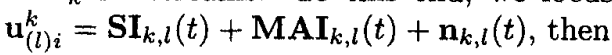

$$
\begin{aligned}
z_{i}^{k}(t)=\sum_{l=1}^{L_{k}} \sqrt{P_{i}^{k}} G_{k, l} & d_{k, i}\left(n T_{b}\right)\left(\mathbf{w}_{(l)}^{k}\right)^{H} \mathbf{a}_{k, l} \\
& +\sum_{l=1}^{L_{k}}\left(\mathbf{w}_{(l)}^{k}\right)^{H} \mathbf{u}_{(l) i}^{k} e^{j \omega_{c} \tau_{k, l}} .
\end{aligned}
$$

Assuming that we use the minimum noise variance criteria to calculate the beamforming/RAKE vector, it can be shown that the optimum weights are[6]

$$
\mathbf{w}_{(l)}^{k}=\frac{R_{\mathbf{u u}_{(l) i}^{k}}^{-1} \mathbf{a}_{k, l}}{\mathbf{a}_{k, l}^{H} R_{\mathbf{u u}_{(l) i}^{k}}^{-1} \mathbf{a}_{k, l}}
$$

where $R_{\mathbf{u u}_{(l) i}^{k}}=E\left\{\mathbf{u}_{(l) i}^{k}\left(\mathbf{u}_{(l) i}^{k}\right)^{H}\right\}$. If the number of antenna elements, $D$, in the antenna arrays is large enough so that we can assume that the effect of $\mathbf{S I}_{k, l}(\cdot)$ in (3) can be neglected, then the signal to noise ratio for the $i^{\text {th }}$ substream of user $k$, is [7]

$$
\gamma_{i}^{k}=\frac{P_{k, i} \Psi^{k}}{\sum_{j \neq k} \sum_{i=1}^{r_{j}} P_{j i} \Phi^{k, j}+N_{k}}
$$

where $\Psi^{k}=L \sum_{l, \check{m}=1}^{L_{k}} G_{k, l} G_{k, \check{m}}^{*}\left(\left(\mathbf{w}_{l}^{k}\right)^{H} \mathbf{a}_{k, l}\right)\left(\left(\mathbf{w}_{\tilde{m}}^{k}\right)^{H} \mathbf{a}_{k, \check{m}}\right)^{*}$ and $\Phi^{k, j}=\sum_{l=1}^{L_{j}} G_{k j_{l}}^{2}\left|\left(\left(\mathbf{w}_{l}^{k}\right)^{H} \mathbf{a}_{k j_{l}}\right)\right|^{2}$, where $L$ is the processing gain.

For simplicity, assume we use PSK, then the probability of bit error conditioned on the SINR is $\operatorname{Pr}\left(\right.$ error $\left.\mid \gamma_{k}\right)=$ $\mathcal{Q}\left(\sqrt{2 \gamma_{k}}\right)$, which we shall denote $p_{e, k}$. Here, $\mathcal{Q}(x)=$ $\frac{1}{\sqrt{2 \pi}} \int_{x}^{\infty} e^{\frac{-t^{2}}{2}} d t$. The channel capacity for substream $i$ of user $k$, given $\gamma_{i}^{k}$ is then $C_{k}=1-H\left(p_{e, k, i}\right)$, where $H(\cdot)$ denotes the entropy due to the probability of error $p_{e, k, i}$. Now, assuming a transmission rate of $\hat{R}_{t}$ for user $k$, the reception rate for that substream of user $k$ is $R_{k, i}=\left(1-H\left(p_{e, k, i}\right)\right) \hat{R}_{t}$.

For each user, $k$, we wish to choose the best number of substreams, $r_{k}$, and for each of his substreams, $i$, we wish to choose the optimum SINR assignment, $\gamma_{i}^{k}$, in order to maximize the total capacity for that $k$. To write an optimization algorithm for the rate over $\left\{r_{k}, \gamma_{i}^{k}, i=1, \cdots, r_{k}\right\}_{k \in I}$, where $I$ is the set of indexes for multimedia users, we identify a cost function, $J$ :

$$
\begin{aligned}
R_{\text {tot }} & =\hat{R}_{t} \sum_{k \in I} \sum_{i=1}^{r_{k}}\left(1-H\left(p_{e, k, i}\right)\right) \\
\Rightarrow \quad J & \triangleq \sum_{k \in I} \sum_{i=1}^{r_{k}}\left(1-h\left(\gamma_{i}^{k}\right)\right),
\end{aligned}
$$

where $h\left(\gamma_{i}^{k}\right)=H\left(p_{e, k}\left(\gamma_{i}^{k}\right)\right)$

The SINR levels that we assign cannot be arbitrarily large. If they are too large, then the power control algorithm breaks down, and we do not get positive powers for all users. In order to guarantee that the power control algorithm works, we must ensure that the target SINR levels we choose are feasible.

The number of all users' substreams is $N=\sum_{k=1}^{M} r_{k}$. Then we define the $N \times N$ matrix $\Gamma$ as a diagonal matrix with matrices $\gamma^{k}$ along the diagonal. Each $r_{k} \times r_{k}$ matrix $\gamma^{k}$ is a diagonal matrix with elements $\gamma_{1}^{k}, \cdots, \gamma_{r_{k}}^{k}$. Let $F$ be the system matrix, which is defined by

$$
F=\left(\begin{array}{cccc}
0 & A_{12} & \cdots & A_{1 M} \\
A_{21} & 0 & \cdots & A_{2 M} \\
\vdots & \vdots & \vdots & \vdots \\
A_{M 1} & A_{M 2} & \cdots & 0
\end{array}\right),
$$




\begin{tabular}{|l|c|}
\hline Requirement & Index Set Name \\
\hline \hline$\mu_{2 i-1}^{*}=0$ and $\mu_{2 i}^{*}=0$ & $A$ \\
\hline$\mu_{2 i-1}^{*}=0$ and $\gamma_{i}^{*}=m_{i}$ & $B$ \\
\hline$\mu_{2 i}^{*}=0$ and $\gamma_{i}^{*}=M_{i}$ & $C$ \\
\hline$\gamma_{i}^{*}=m_{i}$ and $\gamma_{i}^{*}=M_{i}$ & $\emptyset$ \\
\hline
\end{tabular}

TABLE I

POSSIBLE REQUIREMENTS FOR OPTIMAL SOLUTION.

where each matrix $A_{a b}=\frac{\Phi^{a, b}}{\Psi^{a b}} \mathbf{1}_{a b}$, where $\mathbf{1}_{a b}$ is an $r_{a} \times$ $r_{b}$ matrix of ones. Then the SINR levels, $\gamma_{1}^{1}, \cdots, \gamma_{r_{1}}^{1}$, $\cdots, \gamma_{1}^{M} \cdots, \gamma_{r_{M}}^{M}$, are feasible if the spectral radius of $\Gamma F$ has norm less than one, $|\rho(\Gamma F)|<1,[7]$.

Let the $N$-dimensional vector $\vec{\gamma}$ be defined by $\vec{\gamma}=$ $\left(\gamma_{1}^{1}, \cdots, \gamma_{r_{1}}^{1} \cdots, \gamma_{1}^{M} \cdots, \gamma_{r_{M}}^{M}\right)$, and say that we constrain the SINR levels for each substream to be inside some prespecified intervals. Due to the nature of multimedia data, we can assign higher SINR intervals to substreams that require higher rates and lower SINR intervals to those which require lower rates (such as a video substream as opposed to an audio substream). We have shown that the optimization problem:

$$
\begin{array}{ll}
\max _{\vec{\gamma}, r_{1}, \cdots, r_{M}} J & \text { such that } \\
|\rho(\Gamma F)|<1, & \\
\gamma_{i}^{k} \in\left(\gamma_{k, i}^{\text {min }}, \gamma_{k, i}^{\text {max }}\right) \subset \mathcal{R}^{+}, & i=1, \cdots r_{k}, \\
r_{k} \in\left\{1,2, \cdots, r_{\max }\right\}, & k=1, \cdots, M .
\end{array}
$$

is NP-hard [7]. The part that makes this problem intractable is the second line of (9), which is the test for feasibility of $\Gamma$.

Finding the optimal selection of SINR levels, and choosing the optimal number of substreams per user is an optimization problem with two types of variables, real, such as the $\gamma_{i}^{k}$, and integer, such as the $r_{k}$. In general, hybrid integer problems are intractable[8], however, we show that, under certain circumstances, this problem can be broken down into two simpler problems, one of which has an optimal solution and the other has a suboptimal solution which can be reached through methods developed in [9].

\section{A. Optimization Problem is Separable}

Under the assumptions used for (6), the feasibility test for $\Gamma$ in (9) can be simplified. We have shown that testing the feasibility of $\Gamma$ is the same as testing the feasibility for the matrix $\tilde{\Gamma}$, which is a diagonal matrix with diagonal entries $\sum_{i=1}^{r_{k}} \gamma_{i}^{k}$. Since all the interference and noise are the same for the substreams of a given user, it makes sense to talk about the summation of SINR levels.

The reduced system matrix would be the $M \times M$ matrix $\tilde{F}$, which has zeros along the diagonal, and has elements $\tilde{F}_{i j}=\Phi^{k, j} / \Psi^{i}, i \neq j$. There are two advantages of testing for feasibility of $\tilde{\Gamma}$ with $|\rho(\tilde{\Gamma} \tilde{F})|$, as opposed to testing the

\begin{tabular}{|c|c|}
\hline 1. & For each index assignment in $D$, do: \\
\hline 2. & If $A \neq \emptyset$, then \\
\hline 2.a. & $\bar{\gamma}_{\text {new }}=\bar{\gamma}-\sum_{i \in B} \gamma_{i}^{*}-\sum_{i \in C} \gamma_{i}^{*}$ \\
\hline 2.b. & Set $\gamma_{i}^{*}=\gamma_{A}^{*}=\frac{\gamma_{n e w u}}{r_{A}}$ for all $\gamma_{i}$ with $i \in A$. \\
\hline 2.c. & $\begin{array}{l}\text { If } \gamma_{A}^{*} \text { is not in the constraining interval for } \\
\text { some } i \in A \text {, then go to Step } 4 \text {. }\end{array}$ \\
\hline 2.d. & Find $\psi^{*}=\frac{\partial c\left(\gamma_{A}^{*}\right)}{\partial \gamma_{i}}$ \\
\hline 2.e. & $\begin{array}{l}\text { If } \mu_{2 i-1}^{*}=\frac{\partial c\left(M_{i}\right)}{\partial \gamma_{i}}-\psi^{*}, i \in C, \text { is negative, } \\
\text { then go to Step } 4 .\end{array}$ \\
\hline 2.f. & $\begin{array}{l}\text { If } \mu_{2 i}^{*}=\psi^{*}-\frac{\partial c\left(m_{i}\right)}{\partial \gamma_{i}}, i \in B, \text { is negative, } \\
\text { then go to Step } 4 .\end{array}$ \\
\hline 2.g. & $\begin{array}{l}\text { Set all } \gamma_{i}^{*}=m_{i} \text { for } i \in B, \text { and all } \gamma_{i}^{*}=M_{i} \text { for } \\
i \in C \text { and return }\left\{\gamma_{i}^{*}\right\}_{i=1, \cdots, r} \text { Exit. }\end{array}$ \\
\hline 3. & If $A=\emptyset$, then \\
\hline 3.a. & If $\max _{i \in B} \frac{\partial c\left(m_{i}\right)}{\partial \gamma_{i}}>\min _{i \in C} \frac{\partial c\left(M_{i}\right)}{\partial \gamma_{i}}$, then go to Step 4 . \\
\hline 3.b. & If $\sum_{i \in B} m_{i}+\sum_{i \in C} M_{i} \neq \bar{\gamma}$, then go to Step 4. \\
\hline 3.c. & $\begin{array}{l}\text { Set all } \gamma_{i}^{*}=m_{i} \text { for } i \in B, \text { and all } \gamma_{i}^{*}=M_{i} \text { for } \\
i \in C \text { and return }\left\{\gamma_{i}^{*}\right\}_{i=1, \cdots, r} \text {. Exit. }\end{array}$ \\
\hline 4. & $\begin{array}{l}\text { There is no solution for this } D \\
\text { go to the next } D \text { and go to Step } 2 \text {. }\end{array}$ \\
\hline 5. & $\begin{array}{l}\text { If there were no solutions for any combinations } \\
\text { in } D \text {, then there is no solution. Exit. }\end{array}$ \\
\hline
\end{tabular}
feasibility of $\Gamma$ with $|\rho(\Gamma F)|$. The first, most obvious advantage is that $\tilde{F}$ and $\tilde{\Gamma}$ are $M \times M$ matrices, whereas $F$ and $\Gamma$ are $N \times N$ matrices.

\section{TABLE II}

ALGORITHM FOR OBTAINING THE OPTIMAL SET OF $\left\{\gamma_{i}^{*}\right\}_{i=1, \cdots, r}$, GIVEN $r$ AND $\bar{\gamma}$.

The second advantage is that we need not test for the feasibility of the SINR for every single substream of every user, $\left\{\gamma_{i}^{k}, i=1, \cdots, r_{k}\right\}_{k=1, \cdots, M}$. We only need to test for the feasibility of the additive SINR for each user $\left\{\sum_{i=1}^{r_{k}} \gamma_{i}^{k}\right\}_{k=1, \cdots, M}$.

The problem of finding the optimum $\tilde{\Gamma}$ with $|\rho(\tilde{\Gamma} \tilde{F})|$ is NP-hard,[10]. However, we have developed an algorithm that arrives to a suboptimal solution for this problem, [9].

Once we have assigned the additive SINR, $\sum_{i=1}^{r_{k}} \gamma_{i}^{k}$, for each user, $k$, then we must still decide what is the optimum number of substreams for that user, $r_{k}$, and the SINR allocation for each substream, $\gamma_{i}^{k}$. Using constrained optimization theory, we have shown that given $\sum_{i=1}^{r_{k}} \gamma_{i}^{k}$ for user $k$, we can find a globally optimum $r_{k}$ and $\gamma_{i}^{k}, i=1, \cdots, r_{k},[7]$.

\section{B. Optimal $r_{k}$ and $\gamma_{i}^{k}$ for a User}

We can use the algorithms introduced in [9] to find a suboptimal total SINR allocation for each user $k, \bar{\gamma}=\sum_{i=1}^{r_{k}} \gamma_{i}^{k}$. Now, for each user, we need to choose $r_{k}$ and each $\gamma_{i}^{\bar{k}}$.

We proved that when there are no constraints on each individual $\gamma_{i}^{k}$, then the optimal SINR allocation is that all substreams have the same SINR, $\gamma_{i}^{k}=\bar{\gamma} / r_{k}$, for $i=$ $1, \cdots, r_{k},[7]$. And we also showed that the higher the number of substreams, $r_{k}$, the higher the rate. However, this would suggest that the optimal solution for unconstrained $\gamma_{i}^{k}$ is when you have an unlimited number of substreams. 


\begin{tabular}{|c|l|}
\hline 1. & Choose $r=1$ and $I_{1}=\left[m_{1}^{1}, \bar{M}_{1}^{1}\right]$ \\
\hline 2. & $\begin{array}{l}\text { For a sampling of } \bar{\gamma} \in\left[\bar{\gamma}_{\min }, \bar{\gamma}_{\max }\right] \text {, get the } \\
\text { optimum }\left\{\gamma_{r_{i}}^{*}\right\}_{i=1, \cdots, r}, \text { if they exist. }\end{array}$ \\
\hline 3. & $\begin{array}{l}\text { Calculate the total capacity, given } \\
\left\{\gamma_{r_{i}}^{*}\right\}_{i=1, \cdots, r}: \sum_{i=1}^{r} c\left(\gamma_{r_{i}}^{*}\right)\end{array}$ \\
\hline 4. & $\begin{array}{l}\text { Set } r=r+1, \text { and define } \\
I_{1}=\left[m_{1}^{r}, M_{1}^{r}\right], \cdots, I_{r}=\left[m_{r}^{r}, M_{r}^{r}\right] .\end{array}$ \\
\hline 5. & Repeat from Step 2 until $r=r_{\max .}$ \\
\hline 6. & For each $\bar{\gamma}$, compare the resulting $\sum_{i=1}^{r} c\left(\gamma_{r_{i}}^{*}\right)$ \\
\hline 7. & For each $\bar{\gamma}$, choose $r^{*}=\arg { }_{r=1, \cdots, r_{m a x}} \sum_{i=1}^{r} c\left(\gamma_{r_{i}}^{*}\right)$ \\
\hline 8. & Assign $\left\{\gamma_{r_{i}^{*}}^{*}\right\}_{i=1, \cdots, r^{*}}$ to each substream. \\
\hline
\end{tabular}

\section{TABLE III}

ALGORITHM FOR OBTAINING THE BEST $r$ AND THE OPTIMAL SET OF

$$
\left\{\gamma_{i}^{*}\right\}_{i=1, \cdots, r} \text { FOR VARIOUS } \bar{\gamma} \text {. }
$$

This would yield undesirable results for at least two reasons. First, the transmitter and receiver would have to handle a very large number of substreams, which implies a large number of decorrelations, space-time diversity processors, etc. Another reason would be that $\gamma_{i}^{k}$ gets arbitrarily small with arbitrarily high $r_{k}$. Low SINR levels contribute to high bit error rates, which may be handled with very sophisticated coding methods. But these methods require a great deal of computing power, which translate into depleting the battery resources.

It is more reasonable to consider a constraint on $r_{k}$, say we define the maximum number of substreams any user can have, $r_{\max }$, and we limit the range of SINR levels for each substream.

We rewrite Problem (9) for a single user with $r$ substreams, in a format with we can use constrained optimization methods. Let $\gamma=\left(\gamma_{1}, \cdots, \gamma_{r}\right)$, the problem is

$$
\begin{gathered}
\min _{\gamma} f^{0}(\gamma)=-\sum_{i=1}^{r} c\left(\gamma_{i}\right) \quad \text { such that } \\
g(\gamma)=\bar{\gamma}-\sum_{i=1}^{r} \gamma_{i}=0 \\
f^{1}(\gamma)=\gamma_{1}-\gamma_{1}^{\max } \leq 0 \\
f^{2}(\gamma)=\gamma_{1}^{\min }-\gamma_{1} \leq 0 \\
\vdots \\
f^{2 r-1}(\gamma)=\gamma_{r}-\gamma_{r}^{\max } \leq 0 \\
f^{2 r}(\gamma)=\gamma_{r}^{\text {min }}-\gamma_{r} \leq 0
\end{gathered}
$$

It can be shown that if $\gamma^{*}$ is a local minimizer for (10), then there exists a $\mu^{*} \in \mathcal{R}^{2 r}$, and $\psi^{*} \in \mathcal{R}$ such that

$$
\begin{gathered}
\mu^{*} \geq 0 \\
f^{i}\left(\gamma^{*}\right) \leq 0, \quad i=1, \cdots, 2 r \\
g\left(\gamma^{*}\right)=0, \\
\nabla f^{0}\left(\gamma^{*}\right)+\sum_{i=1}^{2 r} \mu_{i}^{*} \nabla f^{i}\left(\gamma^{*}\right)+\psi^{*} \nabla g\left(\gamma^{*}\right)=0 \\
\mu_{i}^{*} f^{i}\left(\gamma^{*}\right)=0, \quad i=1, \cdots, 2 r,[11] .
\end{gathered}
$$

\begin{tabular}{|c|c|}
\hline$r$ & $I_{i}=\left[m_{i}^{r}, M_{i}^{r}\right],[\mathrm{dB}]$ \\
\hline \hline 1 & $I_{1}=[2,30]$ \\
\hline 2 & $I_{1}=[5,30], I_{2}=[2,15]$ \\
\hline 3 & $I_{1}=[7,30], I_{2}=[5,20], I_{3}=[2,15]$ \\
\hline 4 & $I_{1}=[8,30], I_{2}=[7,20], I_{3}=[6,18], I_{4}=[2,15]$ \\
\hline 5 & $I_{1}=[10,30], I_{2}=[9,25], I_{3}=[8,20], I_{4}=[7,18]$ \\
& $I_{5}=[2,15]$ \\
\hline 6 & $I_{1}=[11,30], I_{2}=[10,25], I_{3}=[9,20], I_{4}=[8,18]$ \\
& $I_{5}=[7,16], I_{6}=[2,15]$ \\
\hline 7 & $I_{1}=[12,30], I_{2}=[11,26], I_{3}=[10,25], I_{4}=[9,20]$ \\
& $I_{5}=[8,18], I_{6}=[7,16], I_{7}=[2,15]$ \\
\hline
\end{tabular}

TABLE IV

SINR CONSTRAINTS FOR DIFFERENT NUMBERS OF SUBSTREAMS.

We must find if there are $\mu^{*}$ and $\psi^{*}$, as specified in (11) such that the proper conditions are met. Consider that for each $i=1, \cdots, r$, we have that $\left(\mu_{2 i-1}^{*}=0\right.$ or $\left.\gamma_{i}^{*}=M_{i}\right)$ and $\left(\mu_{2 i}^{*}=0\right.$ or $\left.m_{i}=\gamma_{i}^{*}\right)$. These conditions reduce us to four possibilities for each substream $i$, these are detailed in Table I.

Table II is a method to find the optimum set of $\left\{\gamma_{r_{i}}^{*}\right\}_{i=1, \cdots, r}$, given $r$ and a specific $\bar{\gamma}$. This procedure does an exhaustive search of all possible assignments of the indexes, $i=1, \cdots, r$ to the sets $A, B$, and $C$, as defined in Table I. So, for example, if $r=2$, then we consider all the following combinations for indexes $i=1,2: 1,2 \in A$, $1,2 \in B, 1,2 \in C, 1 \in A$ and $2 \in B, 1 \in A$ and $2 \in C$, etc. We will denote all of these possible combinations as $D$. Finally, to solve our problem for our user, we follow the algorithm outlined in Table II.

\section{SimUlations}

We study the capacity of a single user, given that we defined sets of intervals according to each $r=1,2, \cdots, 7$. The intervals are specified in Table IV. With these intervals, we chose $\bar{\gamma}$ to take on values between $0 \mathrm{~dB}$ to $30 \mathrm{~dB}$. For each set of $\bar{\gamma}$, we plotted the capacity achieved by the optimal solution, if there was one. The resulting graph can be seen in Figure 3. In this graph, negative values of capacity indicate that there was no solution for that number of substreams and that $\bar{\gamma}$.

It should be noted that the shape of the graph in Figure 3 is highly dependent on the choice of intervals. If the system administrator does not wish to allow discontinuities in the capacity for any user, then the intervals should be chosen to reflect that.

We can see in Figure 3 that for $\bar{\gamma}<17.6989 \mathrm{~dB}$, there are no solutions, $\left\{\gamma_{i}^{*}\right\}_{i=1, \cdots, 7}$ for $r=7$. But for $\bar{\gamma} \geq 17.6989 \mathrm{~dB}$, the optimal capacity is achievable with seven substreams. Furthermore, seven substreams yields a higher overall user capacity than any other choice of $r$.

Another example is if we see $\bar{\gamma}=13.5 \mathrm{~dB}$. Here, there are optimal solutions for $r=1,2,3$, and $r=4$ substreams. But for this value of $\bar{\gamma}$, the best capacity is achieved at $r=4$ substreams. 


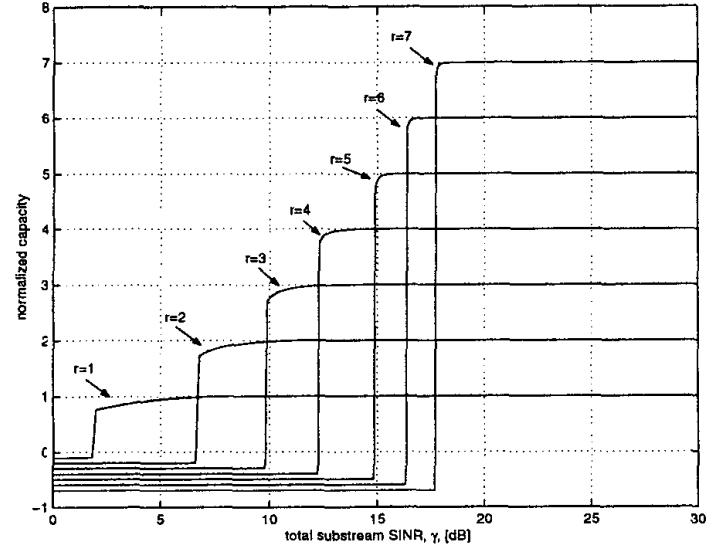

Fig. 3. The optimal number of substreams, $r$, can be seen for each total $\gamma$.

By defining our intervals, $I_{i}$, differently, we obtain different graphs, which in turn, show different thresholds for us to change from one number of substreams to another number of substreams.

In general, a wireless system administrator would have to define a set of thresholds that would satisfy generic rate requirements for different service types, keeping in mind that different users may use different data formats. For example, Two different users may use different video coders, but the system need only identify that these two users both require high rates for their video information.

Once the intervals were defined, we implement the algorithm described in Table II and Table III, and we generate a table that can be referenced on-line by the system. Each time $\varepsilon_{b}$ user is assigned a $\bar{\gamma}$, the system checks the table to check what is the best number of substreams and what $\left\{\gamma_{i}^{*}\right\}$ should be assigned to each substream. Using the intervals from Table IV, we have created a sample look-up table, Table V.

Once that is done, then the method explained in [9] is used to find the optimum power vector and antenna array weights.

\section{Conclusions}

We focus on providing higher data rates for users which are identified as high rate multimedia users. We define a cost function, (7), that depends on the effective SINR levels and the chosen set of substreams for each user.

We use a steepest descent method to find a suboptimal solution for the total SINR assignment for each user,[7], [9]. Once we have each users' SINR allotment, $\bar{\gamma}$, we can optimally choose how many substreams to assign to each user, and what transmission power should be used for each substream. With our method, each multimedia user can separate his data stream into substreams that have distinct rates.

It should be noted that the choice of SINR constraints, such as the example in Table IV has an impact on the overall capacity for the user. The proper choice of intervals

\begin{tabular}{|c|c|c|}
\hline $\begin{array}{c}\bar{\gamma} \\
{[d B]}\end{array}$ & $r$ & $\begin{array}{c}\gamma_{1}^{*}, \cdots \gamma_{r}^{*} \\
{[d B]}\end{array}$ \\
\hline$<2$ & - & no solution \\
\hline $2-6$ & 1 & $\gamma_{1}^{*}=\bar{\gamma}$ \\
\hline 7 & 2 & $\gamma_{1}^{*}=2.6708, \gamma_{2}^{*}=5$ \\
\hline 8 & 2 & $\gamma_{1}^{*}=4.9794, \gamma_{2}^{*}=5$ \\
\hline 9 & 2 & $\gamma_{1}^{*}=\gamma_{2}^{*}=5.9897$ \\
\hline 10 & 3 & $\gamma_{1}^{*}=2.6147, \gamma_{2}^{*}=5, \gamma_{3}^{*}=7$ \\
\hline 11 & 3 & $\gamma_{1}^{*}=\gamma_{2}^{*}=5.7849, \gamma_{3}^{*}=7$ \\
\hline 12 & 3 & $\gamma_{1}^{*}=\gamma_{2}^{*}=\gamma_{3}^{*}=7.2288$ \\
\hline 13 & 4 & $\gamma_{1}^{*}=\gamma_{2}^{*}=6.3504, \gamma_{3}^{*}=7, \gamma_{4}^{*}=8$ \\
\hline 14 & 4 & $\gamma_{1}^{*}=\gamma_{2}^{*}=\gamma_{3}^{*}=7.9725, \gamma_{4}^{*}=8$ \\
\hline 15 & 5 & $\begin{array}{c}\gamma_{1}^{*}=3.7255, \gamma_{2}^{*}=7 \\
\gamma_{3}^{*}=8, \gamma_{4}^{*}=9, \gamma_{5}^{*}=10\end{array}$ \\
\hline 16 & 5 & $\gamma_{1}^{*}=\gamma_{2}^{*}=\gamma_{3}^{*}=8.6268, \gamma_{4}^{*}=9, \gamma_{5}^{*}=10$ \\
\hline 17 & 6 & $\begin{array}{c}\gamma_{1}^{*}=\gamma_{2}^{*}=\gamma_{3}^{*}=8.1483 \\
\gamma_{4}^{*}=9, \gamma_{5}^{*}=10, \gamma_{6}^{*}=11\end{array}$ \\
\hline 18 & 7 & $\begin{array}{c}\gamma_{1}^{*}=\gamma_{2}^{*}=7.1620, \gamma_{3}^{*}=8, \gamma_{4}^{*}=9 \\
\gamma_{5}^{*}=10, \gamma_{6}^{*}=11, \gamma_{7}^{*}=12\end{array}$ \\
\hline 19 & 7 & $\begin{array}{c}\gamma_{1}^{*}=\cdots=\gamma_{5}^{*}=10.0855 \\
\gamma_{6}^{*}=11, \gamma_{7}^{*}=12\end{array}$ \\
\hline 20 & 7 & $\gamma_{1}^{*}=\cdots=\gamma_{6}^{*}=11.4691, \gamma_{7}^{*}=12$ \\
\hline 21 & 7 & $\gamma_{1}^{*}=\cdots=\gamma_{7}^{*}=12.5490$ \\
\hline 22 & 7 & $\gamma_{1}^{*}=\cdots=\gamma_{7}^{*}=13.5490$ \\
\hline 23 & 7 & $\gamma_{1}^{*}=\cdots=\gamma_{7}^{*}=14.5490$ \\
\hline
\end{tabular}

TABLE V

SAMPLE LOOK-UP TABLE.

is a subject for further study.

\section{REFERENCES}

[1] Y. Liang; F. Chin; and K.J.R. Liu, "Downlink Beamforming for DS-CDMA Mobile Radio with Multimedia Services," Proc. of IEEE 50th Vehicular Technology Conference, vol. 1, pp. 17-21, 1999.

[2] F. Rashid-Farrokhi and L. Tassiulas, "Power Control and SpaceTime Diversity for CDMA Systems," IEEE GLOBECOM, vol. 4, pp. 2134-2140, November 1998.

[3] A. Paulraj and C. Papadias, "Space-Time Processing for Wireless Communications," IEEE Signal Processing Magazine, vol 14, no. 6, pp. 49-83, November 1997.

[4] F. Rashid-Farrokhi; L. Tassiulas; and K.J.R. Liu, "Joint Optimal Power Control and Beamforming in Wireless Networks Using Antenna Arrays," IEEE Transactions on Communications, vol 46, no. 10, pp. 1313-1324, October 1998.

[5] A. Naguib, Adaptive Antennas for CDMA Wireless Networks, Ph.D. thesis, Stanford University, August 1996.

[6] R. Monzingo and T. Miller, Introduction to Adaptive Arrays, Wiley - Interscience, 1980.

[7] A. Mercado, Adaptive Service Rate for Integrated Multimedia Over Wireless Networks, Ph.D. thesis, University of Maryland at College Park, July 2001.

[8] C. Floudas, Nonlinear and Mixed-Integer Optimization, Oxford University Press, 1995.

[9] A. Mercado; K.J.R. Liu, "Adaptive QoS for Mobile Multimedia Applications with Power Control and Smart Antennas," IEEE International Communications Conference, vol. 1, pp. 60-64, June 2000.

10] A. Mercado; K.J.R. Liu, "NP-Hardness of the Stable Matrix in Unit Interval Family Problem in Discrete Time," Systems and Control Letters, vol. 42, no. 4, pp. 261-265, April 2001.

[11] A. Tits, Notes for ENEE 664: Optimal Control (draft), August 1998 